\title{
Vivienda y ciudad compacta. Conceptos y debates sobre ecourbanismo en España*
}

\begin{tabular}{|c|c|}
\hline \multicolumn{2}{|c|}{ Fecha de recepción: 4 de febrero de 2016 Fecha de aceptación: 7 de octubre de $2016 \quad$ Disponible en línea: 12 de mayo de 2017} \\
\hline \multicolumn{2}{|l|}{ Sergio Alfonso Ballén Zamora } \\
\hline Magíster en Hábitat y vivienda & Magíster en Arquitectura Sostenible \\
\hline Especialidad Arquitectura y Medio Ambiente & Investigador de la Universidad Nacional de Colombia \\
\hline Universidad Colegio Mayor de Cundinamarca & sergio.ballen@yahoo.com \\
\hline
\end{tabular}

Resumen El urbanismo basado en consideraciones ambientales y sociales ha tenido gran acogida debido a su importancia para la sostenibilidad urbana. Por otro lado, la vivienda no deja de ser una necesidad insatisfecha en muchas ciudades del mundo, lo que afianza un escenario de urbes compactas, consolidadas y con necesidad de expandirse mediante vivienda de alta densidad. Esta investigación identifica definiciones y algunas experiencias desarrolladas en España en las áreas mencionadas en el marco del urbanismo ecológico, con el fin de desarrollar consideraciones cualitativas para la vivienda y el modelo de ciudad que retroalimenten el discurso y proporcionen nuevas luces hacia el diseño de mejores ciudades y viviendas acordes con las necesidades actuales. Se ha escogido el caso español por la buena cantidad de investigaciones del tema, los casos prácticos, las herramientas de evaluación, la facilidad de poder realizar constataciones y el acceso a mayor documentación.

Palabras clave compacta; densidad; urbanismo ecológico; vivienda

\footnotetext{
Artículo de reflexión basado en una investigación con el mismo nombre y presentada en septiembre de 2014 como trabajo de grado para optar al título de máster en Proyecto Avanzado de Arquitectura y Ciudad, Especialidad Arquitectura y Medio Ambiente, de la Universidad de Alcalá (Madrid), como becario de la Fundación Carolina.
}

Cómo citar este artículo: Ballén, S. (2017). Vivienda y ciudad compacta. Conceptos y debates sobre ecourbanismo en España. Cuadernos de Vivienda y Urbanismo, 10(19), 68-85. https://dx.doi.org/10.11144/Javeriana.CVU7-14.vccc 


\section{Housing and Compact City. Concepts and Debates on Eco Urbanism in Spain}

Abstract The planning based on environmental and social considerations has been well received due to its importance for urban sustainability. On the other hand, housing is still an unmet need in many cities around the world, which reinforces a scenario of compact cities, consolidated, and in need of expansion through high-density housing. This research identifies definitions and some experiences developed in Spain in the aforementioned areas, under the framework of ecological urbanism, in order to develop qualitative considerations towards a housing and city model that provides feedback for this discourse and give new insight for the design of better cities and housing in line with current needs. The Spanish case was chosen due to the fair amount of research on the subject, practical cases, evaluation tools, how easy is to corroborate information, and access more documentation.

Keywords compact; density; ecologic urbanism; housing

\section{Moradia e cidade compacta. Conceitos e debates sobre ecourbanismo na Espanha}

Resumo $\mathrm{O}$ urbanismo baseado em consideraçóes ambientais e sociais teve grande recebimento por causa da sua importância para a sustentabilidade urbana. Por outro lado, a habitaçáo continua a ser uma necessidade insatisfeita em muitas cidades do mundo, o que afiança um cenário de urbes compactas, consolidadas e com necessidade de se expandir mediante habitação de alta densidade. Esta pesquisa identifica definiçóes e algumas experiências desenvolvidas na Espanha nas áreas mencionadas no quadro do urbanismo ecológico, a fim de desenvolver consideraçóes qualitativas para a moradia e o modelo de cidade que retroalimentem o discurso e forneçam novas luzes para o design de melhores cidades e moradias acordes com as necessidades atuais. Escolheu-se o caso espanhol pela boa quantidade de pesquisas sobre o tema, casos práticos, ferramentas de avaliação, facilidade para realizar constataçôes e acesso a maior documentação.

Palavras chave compacta; densidade; urbanismo ecológico; habitação 


\section{Introducción}

La vivienda de alta densidad es un elemento implícito cuando nos referimos a un modelo físico de ciudad concentrado (o compacto). La mayoría de la literatura relacionada y de casos de estudio está de acuerdo con que el modelo de ciudad concentrada es la más sostenible, en contraposición con una donde la ciudad crece de manera dispersa, porque consume menos suelo, no se extienden las redes de servicios públicos y la movilidad es más eficiente, entre varias razones. No obstante, el reto de implementarla es grande, sobre todo porque requiere intervenir también la ciudad existente y consolidada, en especial las centralidades, con altas exigencias en transporte, servicios públicos, espacio público y abastecimiento, entre otros.

La vivienda es otro tema de importancia en este modelo, pues las condiciones de habitabilidad y calidad de vida varían según las condiciones urbanas, de confort y de hábitat; por tanto, si la mayoría de las grandes ciudades apuestan por ser compactas, la vivienda, que es donde residen todos sus habitantes, es esencial y tal vez determine su éxito o fracaso.
En este panorama aparece el denominado "urbanismo ecológico", de la mano de importantes expertos con una renovada perspectiva de preservación ambiental y ecológica que incide en la ordenación urbana y que abandona la mirada tradicional en la cual lo ambiental era sinónimo de "paisajismo estilístico" representado con una mancha verde con árboles en fila y áreas libres sin consideraciones de los aspectos climáticos, energéticos y del entorno.

En síntesis, el modelo de ciudad compacto propone vivienda de alta densidad en centralidades urbanas y, dada su importancia, debe ejecutarse también bajo condiciones de sustentabilidad ambiental. Si bien es cierto que la mayoría de las viviendas y edificaciones se construye de acuerdo con la demanda y en suelos disponibles con usos acordes o en edificaciones existentes, es el Estado quien dicta unas directrices mediante el planeamiento urbano y la ordenación del territorio que, en este caso, tiende hacia una mayor consideración del entorno y del ambiente, así como la preservación de los mismos. 


\section{Urbanismo ecológico}

El término “ecourbanismo” se popularizó y convirtió rápidamente en el enfoque contemporáneo de aplicación disciplinar del urbanismo. Aunque se compone de la contracción del concepto "urbanismo ecológico", se pueden encontrar términos similares como "urbanismo sostenible" y otros que, según el autor, se refieren a la escala de aplicación como "ecociudad" y "ecobarrio".

Para Salvador Rueda (2011b), el urbanismo ecológico se diferencia del ortodoxo en cuanto a que posee un sistema de indicadores y condicionantes (restricciones) que se aplican en la eficiencia del sistema o en la habitabilidad y que están determinadas por el contexto y sus escalas de intervención. La eficiencia del sistema urbano se expone bajo una ecuación que es la función guía de la sostenibilidad urbana: $E / n H$, donde $E$ es el consumo de energía, $n$ el número de personas jurídicas y $H$ la diversidad o complejidad urbana. El objetivo es reducir el consumo de energía $(E)$ y aumentar los valores de una estrategia basada en la información y el conocimiento que componen $n$ y $H$, que es lo contrario de lo que sucede bajo la lógica de desarrollo económico dominante, en el cual los territorios compiten por el máximo consumo de recursos.

La habitabilidad urbana está relacionada con la mejora de la calidad de vida y confort de todos los seres vivos en un contexto temporal, cultural y geográfico. La habitabilidad se desarrolla en conjunto con el espacio público, los equipamientos y servicios básicos, la edificación, la cohesión social y la biodiversidad. Rueda relaciona la vivienda, la edificación y la cohesión social en un ámbito más amplio que busca heterogeneidad social y de usos, más allá del comportamiento energético y de la accesibilidad.
El urbanismo ecológico es posible y se valida gracias a una serie de indicadores. Sus cuatro objetivos básicos son: la compacidad, la complejidad, la eficiencia metabólica y la cohesión social; el modelo de ciudad que mejor reúne estas características es la ciudad compacta. Para Rueda (2011b) los principales indicadores son: 1) ocupación del suelo; 2) espacio público; 3) movilidad; 4) diversidad de usos y funciones urbanas; 5) biodiversidad; 6) metabolismo, y 7) cohesión social.

Adicional a ello, este urbanismo se proyecta en tres planos que se desarrollan en altura, en superficie y subterráneo. Estos planos se proyectan en unidades básicas de ordenación territorial como la manzana y la supermanzana (y su propuesta de unidad de $400 \times 400 \mathrm{~m}$ ), con espacios específicos para acumuladores, reservas, salas de almacenamiento y volumetrías precisas o flexibles.

En síntesis, para Rueda (1997) la ciudad compacta es sostenible por su estabilidad, aumento de la complejidad, proximidad, ahorro y aprovechamiento de recursos, competitividad, explotación y la calidad de vida que puede generar. Respecto a la vivienda, los edificios deberían diseñarse con base en la dualidad privacidad-espacio común con mayores áreas y un espacio urbano cotidiano que dé respuesta a la dicotomía soledad-colectividad, con apoyo en las tecnologías de la información y la comunicación (TIC).

El arquitecto Miguel Ruano ${ }^{1}$ (1999) afirma que el urbanismo ecológico "define el desarrollo de comunidades humanas multidimensionales sostenibles en el seno de entornos edificados armónicos y equilibrados" (p. 10). Se trata de un concepto y una disciplina cada vez más tratada por ser una visión "integrada" del urbanismo, su inclusión de las tecnologías de la información y los problemas sociales y ambientales.

Arquitecto y planificador (Barcelona, MIT, Harvard) especializado en diseño y desarrollo de proyectos de gran escala. En su libro sobre urbanismo ecológico (1999) recoge proyectos destacados, entre ellos, algunos ejecutados en España. 
Carlos Verdaguer (2000) explica el urbanismo sostenible como "la búsqueda de factores de equilibrio entre naturaleza y ciudad, entre tradición y progreso, entre procesos globales y procesos locales, entre individuo y sociedad, todo ello sobre un escenario territorial atravesado por flujos de materiales y energía" (p. 67), en busca de equilibrio dinámico u homeostático. Sus criterios son: la conservación de los recursos energéticos y materiales, el reequilibrio entre naturaleza y ciudad, la redistribución de los recursos y servicios sobre el territorio, el desarrollo local, la habitabilidad y la cohesión social.

Sobre la premisa del urbanismo sostenible y los conceptos de ecología urbana y ecoaldea, Verdaguer (2000) considera que la noción de ecobarrio reúne todos los aspectos de la sostenibilidad en el ámbito metropolitano, que piensa la ciudad como un conjunto de piezas interconectadas entre lo local y lo global y tiene como propósito regenerar ecológicamente la ciudad y la gestión integrada de los flujos de energía y materia. Sus rasgos principales son la densidad, la mezcla de usos y el predominio del transporte público, ciclista y peatonal.

Con respecto a la densidad, Verdaguer señala que para cada territorio es necesario establecer los límites sobre los cuales se ganan o pierden valores de sostenibilidad y de calidad ambiental del tejido urbano; luego de obtener estos límites, se deberían mezclar tipologías edificatorias. No obstante, también señala que las tipologías edificatorias más nocivas para el ambiente y el tejido son la vivienda unifamiliar y la torre cuando se desarrollan homogéneamente en grandes extensiones.
Para Ester Higueras García (2006), el planeamiento con criterios de desarrollo sostenible se ajusta a la definición de la Carta europea de ordenación del territorio, en la cual el planeamiento territorial es "una disciplina científica, una técnica administrativa y una política concebida como un enfoque multidisciplinario y global" (p. 130), cuyo objeto es el desarrollo equilibrado y la organización física con equidad en las regiones. A su vez, los planes de ordenación urbana son instrumentos de escala municipal para "ordenar en el tiempo y en el espacio" (p. 130), con participación ciudadana y criterios de optimización ambiental.

Según Higueras (2006), una densidad residencial ideal está entre 50 a $65 \mathrm{viv} / \mathrm{ha}$ y existe la necesidad de relacionarlo con tipologías edificatorias. La tipología de bloque abierto presenta ventajas como la posibilidad de orientación solar, las áreas verdes maximizadas y eficiencia en uso del suelo y de las infraestructuras. Entre los inconvenientes se encuentran la monotonía, las corrientes de viento y el tratamiento de los espacios con sombra.

Acerca de los ecobarrios, Higueras (2009) plantea que son desarrollos urbanos que persiguen los principios de eficiencia, equidad y variedad en el marco del concepto de sostenibilidad ${ }^{2}$ y tienen diez temas clave: estudio de la densidad urbana, complejidad y variedad morfológicas, usos mixtos, diversidad socioeconómica, zonas verdes y espacios libres, optimización de redes, equipamientos integrados, gestión del tráfico urbano, reducción de residuos sólidos y valoración del suelo periurbano. 
El enfoque "ecológico" (aunque suene como una redundancia) del urbanismo y la ordenación del territorio aplicado por Feria y Ramos (2009) tiene características interesantes, quizá debido a su formación de geógrafos. En este enfoque, la ciudad es vista como un paisaje ecológico heterogéneo y complejo o bien como un mosaico de hábitats, que es la unidad de análisis desde una visión territorial o de ecología del paisaje. Esta última es definida como "el estudio de las interacciones entre patrones paisajísticos y procesos ecológicos, y más específicamente, de la influencia de dichos patrones en los flujos de agua, energía, nutrientes y biota", cuya aplicación en el ámbito metropolitano resulta de especial utilidad.

Los autores definen espacio libre y estimulan de forma explícita su funcionalidad ambiental como matriz biofísica del territorio, aplicado en hábitats urbanos caracterizados por ser fragmentarios, soportar altos impactos antrópicos y tener presencia de especies invasoras. Es importante la coordinación responsable de los servicios ecológicos, que constituyen el conjunto de beneficios que la sociedad obtiene de los ecosistemas, ya sean bienes o servicios como la regulación del clima, regulación de la composición atmosférica, formación de suelos, control de procesos hidrológicos, mantenimiento de recursos genéticos, etc. (Feria y Ramos, 2009).

A manera de síntesis, podemos afirmar que el urbanismo ecológico es un campo de conocimiento de aplicación en el territorio y paisaje para convertirlo en un sistema homeostático y ordenado, de acuerdo con las interacciones y los impactos entre seres vivos, los aspectos sociales, las infraestructuras y factores abióticos locales y globales en continuo desarrollo. Es preciso tener en cuenta la calidad de vida, la equidad, la

Figura 1

El urbanismo de los tres niveles

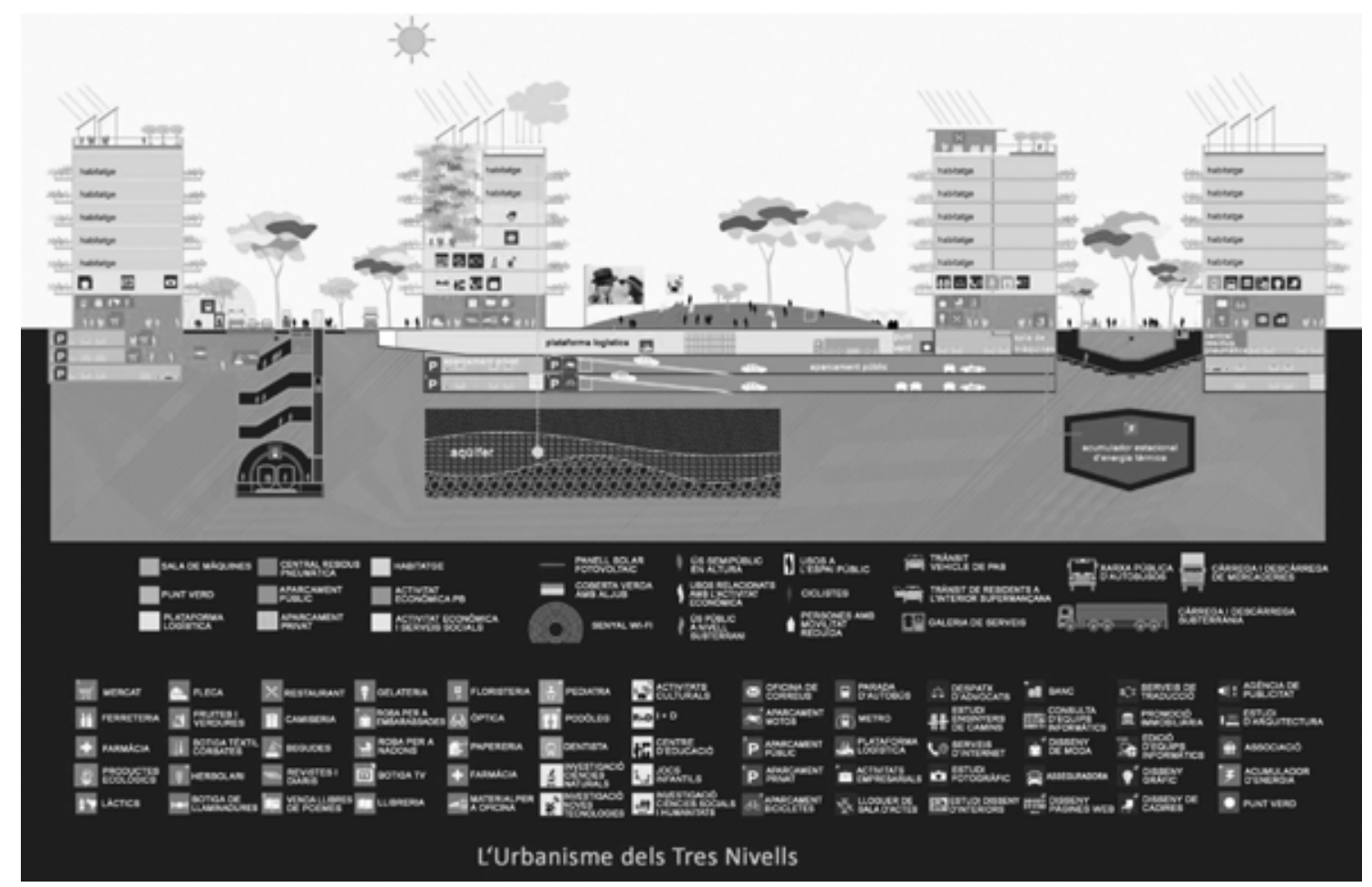

Fuente: Rueda (2011a) 
heterogeneidad, el acceso a la información, el espacio público, la reducción y la eficiencia de recursos, energía y materiales.

Este campo de conocimiento incorpora conceptos vigentes sobre desarrollo sostenible e incentiva la transdisciplinariedad. Carlos Verdaguer (2000) habla de una búsqueda de equilibrio mutualista entre el ser humano, el ambiente y sus elementos; Ester Higueras (2006) presta mayor atención a la noción de desarrollo urbano sostenible y su aplicación en la gestión de infraestructuras; Salvador Rueda (1997) incorpora el acceso a la información, una ecuación de eficiencia metabólica y propone un urbanismo que piensa en tres niveles simultáneos y, por último, José Feria y Jesús Ramos (2009) aplican un enfoque mucho más profundo de la ecología y el territorio, en el que la ciudad es un mosaico ecológico y de altos valores antrópicos.

Vemos también que existe un consenso acerca de que el modelo urbano que mejor se acopla a las características del urbanismo ecológico es la ciudad compacta, debido a particularidades como la proximidad, la heterogeneidad física y social, la ocupación del suelo, la movilidad y la mezcla de usos. Sin embargo, cada una de ellas no es suficientemente explorada en cuanto a sus características y requerimientos; se anticipan como ventajas cualitativas, pero no se consideran las nuevas necesidades ante una ciudad que se compacta masivamente.

\section{Ciudades compactas}

La adopción de un modelo de crecimiento urbano forma parte de la otra cara de la discusión de los planificadores, que trasciende los temas relacionados con la vivienda debido a sus implicaciones en la estructura económica y social para la ciudad. El modelo de "ciudad dispersa" o "interciudad" consiste en la disposición de terrenos sin urbanizar como mecanismo de expansión y crecimiento físico, lo cual conlleva desconcentración de la población, extensión de servicios, "ciudad sin centro" $y$ trabajo en red. Este prototipo tiene objetivos afines con la "ciudad jardín" y procura preservar las bajas alturas edilicias, sobre todo en los centros urbanos por su legado histórico.

Por su parte, el modelo de ciudad "compacta" o "concentrada" se basa en la reutilización de áreas ya urbanizadas, con la incorporación de edificaciones de alta densidad edilicia y demográfica para aprovechar al máximo el suelo como alternativa de crecimiento e involucrar operaciones de renovación o rehabilitación en las zonas centrales deterioradas. Este esquema parte de una conciencia planificadora por la transformación del territorio mediante proyectos de renovación urbana, pero por sus características operativas casi siempre excluye a la población de bajos ingresos de este desarrollo; no obstante, se ha convertido en la directriz de la planificación urbana contemporánea (Rincón, 2006).

La actual compactación de muchas ciudades está ligada a diversos proyectos de densificación, puesto que es un fenómeno que "masifica" la edificación de alta densidad o en altura y es el conector con el modelo de ciudad compacta. La arquitecta Patricia Rincón Avellaneda ${ }^{3}$ (2006) identifica y diferencia los términos “densificación” y "re-densificación”, que son procesos clave relacionados con las variaciones de la densidad poblacional producto de la intensificación del uso del suelo por medio de edificaciones de alta densidad, de acuerdo con diferentes modalidades de desarrollos especulativos del mercado formal e informal.

Esto significa que:

[...] redensificar implica intervenir en algo ya construido, mientras que densificar no necesariamente conlleva este tipo de intervención, puede darse mediante 
operaciones de llenado de áreas vacías al interior de una ciudad.

La redensificación se lleva a cabo de dos maneras, la primera como política urbana de iniciativa pública utilizando generalmente el instrumento de la renovación urbana o las normas, de esta forma se demuele lo existente y se plantean nuevas estructuras con uso más intensivo que el inicial. La segunda como proceso de iniciativa individual con interés privado, se lleva a cabo a través de procesos de subdivisión y con frecuencia saturación de la construcción existente (Rincón, 2006, p. 19).

Para Navarro y Ortuño (2011), la ciudad compacta no puede ser un modelo generalizado, sino una imagen interpretativa de una realidad espacial concreta en el cual la densidad residencial es una variable relevante. Este concepto tiende a considerarse opuesto al análisis de la ciudad difusa y a legitimar un discurso armado de propuestas urbanas con enfoque de sostenibilidad que a veces es contradictorio.

Dicho modelo de crecimiento urbano suele ser vinculado con la ciudad mediterránea tradicional, que busca ser densa, con mezcla de usos y que está vigente por estar asociada con las estrategias de sostenibilidad ambiental urbana a cargo de investigadores relevantes. Navarro y Ortuño (2011) concluyen que las nociones de ciudad compacta se pueden agrupar en cuatro líneas: densidad e imagen interpretativa del territorio, densidad y urbanidad, densidad y eficiencia económica y densidad y eficiencia energética. De esta manera, se relacionan coeficientes de densidad demográfica y de posible eficacia energética enfocada en la movilidad.

Otro interesante punto de vista lo proporciona José Luque (2011), quien señala que el término se refiere a un "punto medio" entre la ciudad dispersa y la urbanización densificada propia de las ciudades de países desarrollados. El autor afirma que escoger cualquier esquema es un acto mecánico que desconoce los ámbitos de convivencia donde se desenvuelve el ser humano, así como la estructura interna, el vínculo entre el espacio edificado y el espacio libre y entre la ciudad y la región, es decir, no existe un desarrollo sostenible real que trascienda la disminución de impactos ambientales.

En su texto sobre la insuficiencia de la ciudad compacta, Luque se orienta a cuestionar el término tradicional "desarrollo sostenible" y propone un enfoque que afronta, en primer lugar, el crecimiento de la persona humana en sus valores familiares, sociales y culturales, lo que supone una revolución ética que proporcione una renovada actitud de esta frente a la naturaleza. En la planificación y el diseño urbano, se busca que la forma urbana posibilite los hábitos ciudadanos que favorecen los dobles principios de "interdependencia-solidaridad" y "pluralismo-complementariedad".

Para comparar las huellas ambientales de los dos modelos de ciudad, Molini y Salgado (2012) analizan la bibliografía disponible que evalúa sus impactos como el consumo de suelo, de energía y de agua, los derivados del transporte, la contaminación y otros. Concluye que todos los estudios y la gran mayoría de autores apoyan el modelo de ciudad compacta como el menos consumidor de recursos. Para hacer las comparaciones, se evaluaron los consumos por hectárea de terreno de viviendas unifamiliares aisladas, unifamiliares adosadas y viviendas en bloque o plurifamiliares como tipologías edilicias residenciales. Además, enfatizan que:

[...] no se debe fomentar cualquier ciudad compacta, sino aquella que, además de generar beneficios ambientales, sea capaz de proporcionar una elevada calidad de vida a sus habitantes. Todas las medidas que favorezcan la ciudad compacta deben ir acompañadas de actuaciones que hagan más confortable el vivir en ella, 
como dotarla de un excelente transporte público y de una adecuada proporción de espacios verdes.

A manera de síntesis de todos los puntos de vista, la ciudad compacta es un modelo interpretativo y de proyección territorial de crecimiento urbano que estimula construcción de edificaciones de alta densidad ${ }^{4}$ e implica procesos de densificación y redensificación, con diferentes escenarios y condiciones de acuerdo con el contexto de aplicación. Su objetivo es reducir impactos ambientales, proporcionar una elevada calidad de vida y tener las condiciones para el disfrute, la convivencia y el confort del ser humano.

Esta definición se basa en las investigaciones consultadas y que es susceptible de ser modificada a la luz del enfoque ambiental del urbanismo ecológico. Es notable también que el modelo urbano tiene en cuenta el transporte público y una buena proporción de espacios verdes respecto al espacio edificado.

La vivienda de alta densidad y los procesos densificatorios en la ciudad conducen a una política de desarrollo que se denomina de aprovechamiento o racionalización del uso del suelo. En ese sentido, es pertinente indagar sobre las políticas y la formación de precios del suelo urbano, su accesibilidad para los sectores de escasos recursos y la generación de plusvalías como temas de alta vigencia en el desarrollo urbano.

Cuando este modelo de ciudad se implementa con el respaldo de la política pública, se ejecutan acciones privadas de aprovechamiento del suelo urbano, se forman nuevas centralidades y se intensifica el uso del suelo por medio de proyectos de densificación y redensificación, con la construcción de vivienda de alta densidad en zonas residenciales, edificios de oficinas en áreas productivas centrales o mixtas. El papel de la vivienda en este modelo de ciudad es esencial, debido a las demandas actuales y futuras de servicios, equipamientos, infraestructuras y movilidad.

\section{Vivienda}

De acuerdo con la bibliografía consultada, no fue posible encontrar una definición de vivienda que se acople a las características particulares de la alta densidad. No obstante, es posible configurar una basada en las definiciones generales de algunos autores. Edwin Haramoto (1983) sostiene:

Para estudiar el problema habitacional es necesario enfocar la vivienda desde una perspectiva amplia. La vivienda no solo es la casa, sino que constituye un conjunto de servicios habitacionales que comprende inseparablemente el suelo, la infraestructura y el equipamiento social-comunitario, junto al techo, refugio o casa.

Esta consideración enfatiza la necesidad de servicios y de suelo, que son importantes debido a sus escasez y, por tanto, una de las razones de la densificación de la vivienda.

Podría complementarse con la noción evidenciada por Fabio Giraldo (2004) en la que la casa es un símbolo que permite la existencia del ser humano manifestándose en varias esferas de la sociedad:

Más allá de la ocupación física de un territorio, es un referente simbólico de la existencia humana en el que intervienen las dimensiones esenciales de la sociedad: política, económico-social, y estético-cultural [...]. Escenario para el despliegue de los aspectos individual y social del ser humano, manifiestos en la posibilidad de habitar en el sentido tanto en lo privado - lo íntimo- como de lo público - el mercado-y lo público-público -las instituciones- (p. 41).

Haramoto (1983) no asume la vivienda como una unidad autosuficiente aislada, sino como un producto incidido por infraestructuras, servicios, suelo y procesos complejos de múltiples actores 
y factores circunstanciales que la llevan a cierta evolución en diversas fases, de manera análoga a la del hábitat y en el marco de una propuesta de intervención que busca solucionar integralmente el problema del déficit habitacional. El Programa de las Naciones Unidas para el Desarrollo y UN Hábitat consideran la vivienda como representación simbólica de una cultura y su posibilidad de manifestarse diversamente en lo público y en lo privado, lo que amplía su concepción a la de hábitat e implica una articulación de procesos que requiere de cierta comprensión para ser tratados (Giraldo, 2004).

De acuerdo con las definiciones ya descritas, el estudio sobre la vivienda de alta densidad debe abordarse como una comprensión de sinergias (económicas, políticas y socioculturales) que inciden sobre la unidad de vivienda y su ambiente, las cuales son producto de la necesidad de suelo, infraestructuras, servicios, actividad pública e individual y sus representaciones 5 . Con ello se logra una significación en los habitantes que se manifiesta en una cultura y tiempo determinados. Este conjunto de fenómenos está antecedido por condiciones urbanísticas, económicas y sociales preexistentes. La vivienda de alta densidad se torna en una unidad básica residencial y territorial y uno de los elementos que configuran una ciudad compacta.

Las características propias de estas construcciones la convierten en un modelo distintivo para la convivencia colectiva, la movilidad y el desarrollo urbano ante los requerimientos actuales. Dada su morfología particular (el bloque lineal), surgen acepciones por parte de urbanistas contemporáneos que la denominan como "multifamiliar", "unidad vecinal", "unidad de habitación" o "bloque de apartamentos".

Con base en esto, al continuar la búsqueda de una definición acorde con la investigación, se encontró que el más cercana es la de "multifamiliar", que la Real Academia Espańola (2014) expone así: "Dicho de un edificio: de varias plantas, con numerosos apartamentos, cada uno de los cuales está destinado para habitación de una familia”.

En general, la mayoría de literatura consultada determina la vivienda multifamiliar dentro de una tipología urbana residencial, con características morfológicas especiales por la forma como se ubica, como se agrupa en un predio y dentro de una edificación como apartamentos. Por vivienda de alta densidad entendemos a la vivienda en altura, colectiva o multifamiliar, ya que representa alta densidad demográfica. Así, podemos determinar que la vivienda de alta densidad se caracteriza por: a) la altura total de la edificación residencial; b) el número de pisos o plantas arquitectónicas; c) el número de unidades de vivienda en un área determinada, y d) el volumen de densificación demográfica que genera.

Existe una estrecha relación entre las altas densidades y el intensivo aprovechamiento del suelo en la construcción, traducido en construcciones de varias plantas. Sin embargo, no es lo mismo vivienda de varias plantas o en altura que de alta densidad, porque es posible que existan viviendas unifamiliares y de altas densidades, algo recurrente en asentamientos de origen informal.

Sería útil obtener una definición y las características de lo que es una vivienda en de alta densidad sostenible para que sea posible compararla con las del urbanismo ecológico. Son muy pocas las nociones que se pueden encontrar para el término de "vivienda sostenible", porque se considera dentro de otras más abiertas y generales como "edificación sostenible", "construcción sostenible" o "arquitectura sostenible".

Por ejemplo, para Pablo Iglesias (2010), la vivienda sostenible es un "lugar cerrado y cubierto construido para ser habitado por personas, y capaz 
de mantenerse por sí mismo a nivel económico, social y ecológico" (p. 7), en consideración de parámetros como una parcela sostenible, eficiencia del agua, energía y atmósfera, materiales y residuos y calidad ambiental interior. Desde el punto de vista de un representante inmobiliario, para lograr este tipo de vivienda es necesario tener en cuenta componentes clave como orientación, sistemas de bajo consumo energético, aislamiento térmico, uso de energías renovables e integración con zonas verdes; en general, se trata de adaptar las viviendas a la regla de las tres " $R$ ": reducir, reutilizar y reciclar ("Los cinco imprescindibles", 2013).

Luis Delgado (2005) señala criterios a implementarse en la construcción de viviendas sostenibles, como la adaptación específica al entorno, métodos de calefacción pasivos, selección de los sistemas activos menos consumidores o basados en energías renovables, utilización de materiales reciclables que no generen residuos tóxicos y adecuación del proceso constructivo a elementos sostenibles en cuanto a materias primas, consumos energéticos y producción de residuos.

Entonces, una vivienda sostenible es una construcción en condiciones de habitabilidad y confort interior que utiliza energías renovables, gestiona y recicla materiales y residuos, se integra ecológicamente con el espacio, considera aspectos económicos y sociales de sus habitantes y del entorno y cuenta con sistemas de eficiencia y bajo consumo energético y de agua.

Esta definición sintética no tiene en cuenta los rasgos vistos en las definiciones de vivienda, pero sí tiene mucho en común con las de urbanismo ecológico. Podríamos decir que la vivienda ya no es tratada como una conceptualización abstracta, sino como unidad habitable que incorpora una serie de prestaciones vinculadas con la sostenibilidad, con menor impacto sobre el entorno.
Recordemos que, para los promotores del urbanismo ecológico, la vivienda se subdivide en diversas tipologías edificatorias (unifamiliar, multifamiliar densidad media y alta o torre) que es necesario distribuir de manera heterogénea en el tejido urbano, cuya articulación con el espacio público y la cotidianidad es fundamental para lograr cohesión y diversidad social y de usos.

\section{Cartillas y guías institucionales}

Mediante el planeamiento urbano y la ordenación del territorio, el Estado establece unas directrices para el crecimiento urbano en busca de calidad de vida y desarrollo social. En este caso, son relevantes las consideraciones frente al entorno, a los recursos, a la energía y al ambiente; como en toda actuación urbana, el Estado tiene un papel protagónico en la implementación, el seguimiento y la difusión del urbanismo ecológico y de la ciudad compacta.

Con el fin de identificar las recomendaciones generales desde las instituciones públicas en España en los temas de urbanismo ecológico y vivienda, se hace una breve descripción de los manuales y estudios relacionados y su manera de abordar la vivienda. Para ello, se han buscado todos los manuales o las guías que tuvieran dentro su título palabras relacionadas con el urbanismo sostenible; aquellos documentos enfocados a tratar temas de vivienda o movilidad no fueron tenidos en cuenta ${ }^{6}$.

Los documentos encontrados son:

Estudi de criteris ambientals per a la redacció del planejament urbanistic. Generalitat de Catalunya. Programa de Sostenibilidad Ambiental Urbana Ciudad 21. Cataluña, 2003. Este 
manual catalán establece criterios urbanísticos sobre ocupación del suelo, ciclo del agua, calidad del aire, condiciones acústicas, lumínicas y electromagnéticas, gestión de materiales y de residuos, conservación de la biodiversidad y del patrimonio natural y calidad del paisaje. No se destaca la vivienda como un tema particular, pero sí aboga por un modelo de crecimiento urbano compacto.

Guia de buenas prácticas de planeamiento urbanistico sostenible. Federación de municipios y provincias de Castilla-La Mancha. Colegio de Arquitectos de Castilla-La Mancha. Colegio de ingenieros de caminos, canales y puertos-demarcación de CLM, 2004. No existe un capítulo dedicado a la vivienda, pero sí define pautas de diseño urbano enfocado a promover densidades medias, tipologías con doble fachada y patio de manzana o espacio libre como una alternativa eficiente y económica.

Manual para la redacción de planeamiento urbanístico con criterios de sostenibilidad. Sociedad Pública de Gestión Ambiental, 2005. Además de proponer una serie de recomendaciones en el ámbito urbano, este manual también señala unos objetivos e indicadores que se aplican a los recursos y residuos (en varios escenarios), movilidad, vivienda, rehabilitación, renovación, medio rural y natural e integración social. Los objetivos generales de la vivienda son garantizar el acceso a una vivienda digna para todos, como necesidad básica y primer eslabón en la relación con la ciudad y contribuir desde la planificación de la vivienda a la sostenibilidad global de los tejidos urbanos, para lo cual se proponen criterios $^{7} \mathrm{e}$ indicadores.

Estrategia española de desarrollo sostenible. Ministerio de la Presidencia, 2007. Es el plan de trabajo y áreas de desarrollo relevantes designadas por el Gobierno espańol como base de su desarrollo, articulado en tres ejes: sostenibilidad ambiental, social y global. Estos núcleos albergan una serie de objetivos, entre los que se destacan la conservación y gestión de los recursos naturales y ordenación del territorio, el cambio climático, el empleo, la cohesión social y pobreza y la cooperación internacional. Se trata de un documento público en donde se indican las líneas de acción de desarrollo nacional y repercuten en la planificación urbana y la vivienda.

- Guia del planeamiento urbanistico energéticamente eficiente. Instituto para la Diversificación y Ahorro de la Energía (IDAE) y la Fundación Privada Institut Ildefons Cerdà. 2a edición, 2007. El documento hace recomendaciones acerca de la planificación urbana que logren eficiencia energética, como distribución de espacios libres, parcelación, trazado de vías, forma de edificios y control climático. Expresa los beneficios de no expandirse de manera difusa y la vivienda se trata como un uso urbano cuyas recomendaciones están recogidas dentro de los temas ya enunciados.

Guía para la elaboración del informe de sostenibilidad ambiental de los planes generales municipales en la comunidad autónoma de La Rioja. Gobierno de La Rioja, 2007. Este texto establece una metodología de elaboración y presentación de un informe de sostenibilidad ambiental del Plan general municipal para la comunidad autónoma de La Rioja dentro de de un planeamiento urbanístico sostenible. Ofrece una serie de parámetros a ser desarrollados y ser objeto de seguimiento. El tema de la vivienda no es explícito, pero se muestran criterios para la rehabilitación ambiental y social de zonas degradadas. 
- Buenas prácticas en arquitectura y urbanismo para Madrid: criterios bioclimáticos y de eficiencia energética. Subdirección General de Planificación General y Periferia Urbana del Área de Gobierno de Urbanismo y Vivienda del Ayuntamiento de Madrid y Universidad Politécnica de Madrid, 2009. Es un manual detallado de aplicación en la ciudad de Madrid, ya que se basa en factores climáticos y en características arquitectónicas particulares. En su capítulo "Buenas prácticas bioclimáticas y de eficiencia en la escala urbana" manifiesta que tanto el modelo difuso como el compacto son perjudiciales si son llevados al extremo y propone posibles densidades brutas y netas a ser aplicadas en algunas tipologías de edificación en las que se asume implícita la vivienda.

- Libro verde del medio ambiente urbano. Ministerio de Medio Ambiente, Medio Rural y Marino. Agencia de Ecología Urbana de Barcelona, 2009. Este documento analiza los problemas y las tendencias en varios ámbitos relacionados y formula directrices enfocadas a un urbanismo sostenible. Las áreas trabajadas son la energía, el agua, el uso de recursos y la gestión de residuos, del aire, del ruido urbano y lo rural-urbano. La vivienda es un elemento objeto de las recomendaciones acerca de estos ámbitos que se divide en varias tipologías residenciales.

- Libro blanco de la sostenibilidad en el planeamiento urbanistico español. Ministerio de Vivienda. Gobierno de España, 2010. Este libro aborda la sostenibilidad y referencia manuales y cartillas desarrollados por comunidades autónomas. Entre las recomendaciones sobre vivienda encontramos la rehabilitación y complejidad del patrimonio inmobiliario desocupado o secundario, la reducción del consumo de suelo y las densidades sobredimensionadas.
Guia para el desarrollo sostenible de los proyectos de urbanización. Grupo Sprilur. Gobierno vasco. Barcelona, junio de 2010. Se trata de una metodología y procedimientos de valoración con base en fichas referentes a la sostenibilidad en proyectos de urbanización y que dependen de la tipología de las edificaciones objeto.

- Guía metodológica para los sistemas de auditoría, certificación o acreditación de la calidady la sostenibilidad en el medio urbano. Gobierno de España. Agencia de Ecología Urbana de Barcelona, 2012. Esta guía hace un estudio de diferentes certificaciones en sostenibilidad urbana, con el fin de proponer metodología, indicadores y condicionantes de auditoría o acreditación de alta calidad con énfasis en las ciudades mediterráneas. En el subcapítulo denominado "La compacidad y funcionalidad", defiende que la ciudad compacta sea un eje de aproximación física del territorio al determinar sus infraestructuras y sistemas, en donde la calidad del espacio público desempeña un papel estructural en su sostenibilidad. En cuanto a vivienda, enfatiza en el acceso y promueve la rehabilitación y diversificación de vivienda social ${ }^{8}$ con una buena localización y espacio público.

- Evaluación ambiental del planeamiento urbanistico: guía para el promotor de planes generales de ordenación urbana. Gobierno de Cantabria, s. f. Describe una metodología y los pasos para desarrollar evaluaciones ambientales por agentes interesados. Comienza por el análisis ambiental, la identificación y valoración de efectos sobre el ambiente y termina con un programa de medidas correctoras, de acuerdo con los requerimientos de tramitación del Plan general de ordenación urbana. Debido a que la finalidad es hacer una evaluación ambiental

8 Se prevé que un mínimo de $30 \%$ de la superficie construida residencial se destinará a vivienda social. Por otro lado, el indicador de vivienda tiene como objetivo "Evaluar las necesidades de vivienda existentes en el municipio objeto de la actuación urbanística y los déficits de acceso a la vivienda en el mismo, de manera que la provisión y el tipo de vivienda de la actuación urbanística responda de la forma más adecuada a las necesidades existentes". 
de un territorio, la vivienda es vista como un cuantificable para gestionar los indicadores.

\section{Conclusiones y prospectiva}

El urbanismo ecológico lleva a la ordenación del territorio y del paisaje a pensar más en los impactos ambientales, en equidad social, en la integración estable y ordenada con los ecosistemas y en la coordinación del intercambio eficiente de materias y energía en diferentes escalas, de acuerdo con la calidad de vida, la heterogeneidad, el acceso a la información y el espacio público.

La ciudad compacta es el modelo de crecimiento urbano que mejor se acopla a estas características, ya que permite proximidad, diversidad edilicia y social, aprovechamiento del suelo, movilidad reducida y mezcla de usos. No obstante, se requieren mayores estudios que aclaren su implantación en un escenario futuro y la adaptabilidad de los servicios y las infraestructuras a un uso de mayores proporciones.

Si se tiene menos suelo para urbanizar, la mayor demanda y los altos precios pueden llegar a hacer que las viviendas nuevas sean inasequibles, en especial las de primeras plantas, porque tienen mayores posibilidades de uso. La propuesta de Salvador Rueda sobre el urbanismo de tres niveles parece interesante por las ventajas y desventajas que podría tener el uso intensivo del subsuelo y en altura. A pesar de no ser una solución completa, la rehabilitación de viviendas también parece una buena alternativa, así como mantener un porcentaje de esta destinado a vivienda social.

Las altas demandas de movilidad, servicios y espacio público también podrían llevarse al subsuelo y en altura; sin embargo, en la práctica, en ciudades muy desarrolladas y construidas es difícil visualizar tal aplicación. Las investigaciones analizadas enfatizaron en que la ciudad compacta es un modelo interpretativo y de proyección territorial que consta de edificaciones de alta densidad con diferentes escenarios posibles de acuerdo con el contexto.

Ese escenario o imagen aún no está claro, lo cual podría entorpecer su aplicación' ${ }^{9}$. Tal vez esta imagen es imposible de determinar porque, si bien existen planes de ordenación territorial que instauran un modelo, es el mercado inmobiliario y sus intereses los que determinan la evolución y el desempeño de tal esquema; además, el suelo disponible es fundamental y puede hacer más barato redensificar la ciudad construida que densificar con nuevas construcciones en suelo periférico disponible.

Los conceptos sobre vivienda de alta densidad sostienen que esta consta de una agrupación de unidades residenciales que, junto con su entorno, es influenciada por particularidades económicas, ambientales, políticas y socioculturales $y$, en especial, por necesidad de suelo, infraestructuras, servicios y la actividad humana pública e individual. Por sus características, el bloque residencial se ha convertido en una unidad de modulación territorial contemporánea, ícono de la convivencia colectiva y configurador de la ciudad compacta.

A su vez, la vivienda sostenible es una construcción residencial que procura la implementación de energías renovables, recicla y cuenta con sistemas de eficiencia energética y de recursos, así como con aspectos económicos, sociales y de confort. Esta definición plantea una diferencia sustancial respecto al anterior concepto amplio de vivienda, pues este resalta la actividad sociocultural, las infraestructuras y la necesidad de suelo, mientras la vivienda sostenible tiene una mirada

9 Plantear una imagen física como guía podría ser un buen ejercicio de planificación, aunque limitado, porque no da cuenta del metabolismo urbano, lo socioeconómico, lo ambiental, lo cultural, etc. Algunas imágenes de Le Corbusier o de Ludwig Hilberseimer a mediados del siglo XX dieron una idea de sus planteamientos, a veces venerados, otras veces fuertemente criticados 
centrada en la unidad residencial y sus prestaciones de eficiencia y reciclaje al servicio de la sostenibilidad ambiental.

Las nociones relacionadas con el urbanismo ecológico abarcan mejor estos factores, pues incorporan la integración con los ecosistemas, la calidad de vida, la equidad y el acceso a la información y al espacio público. Muchos de estos elementos podrían parecer obvios, si se tiene en cuenta la escala de aplicación del urbanismo sostenible respecto a la vivienda, pero algunos de ellos podrían llevarse al diseño de unidades residenciales.

No obstante, a pesar de las tendencias de los conceptos, lo que puede hacer que una vivienda se desarrolle dentro de un urbanismo sostenible es la visión y aplicación por las Administraciones gubernamentales y la asimilación por los promotores inmobiliarios y la población. Buena parte de estas iniciativas se ha materializado en diversos proyectos con apoyo de guías institucionales de difusión, pero nunca en planes de ordenación territorial.

Si bien en España se han impulsado procesos de diferente naturaleza ${ }^{10}$, la gran mayoría no ha podido ejecutarse a plenitud, debido a la crisis financiera de los últimos años; sin embargo, diversas fuentes electrónicas destacan los ecobarrios de Valdespartera (Zaragoza), de Logroño y de Figueres (Catalunya) como los más emblemáticos (Figura 2).

En estos proyectos predominan los bloques de viviendas como planteamiento residencial, lo que da cuenta de su papel dentro del urbanismo ecológico en la búsqueda de compacidad y de su capacidad de ser módulo de diseño y ocupación territorial. Por otro lado, la implementación de vivienda de alta densidad también corresponde a una política mundial y de mercado para el aprovechamiento de uso del suelo y evitar la expansión de los límites urbanos.

Para los expertos en urbanismo ecológico y las guías institucionales de promoción, la vivienda es vista como un uso que consta de diferentes tipologías edificatorias y urbanas. Entre ellas sobresale el bloque, cuyo planteamiento debe hacerse a partir de características climáticas, eficiencia energética y gestión de residuos.

El ámbito sociocultural no ha sido ampliamente abordado; a veces, se limita a una proposición participativa y se subestiman algunas causas de la densificación demográfica como la generación de nuevos hogares y la evolución de los hábitos sociales y familiares.

No existe claridad frente a la dicotomía entre buscar mayores densidades mediante la redensificacion como parte del crecimiento urbano en contextos ya urbanizados por promotores inmobiliarios y ciudades que procuran preservar e incrementar los rasgos naturales del entorno y prestar atención a cualidades climáticas y sociales. Esta ambigüedad se debe, en parte, a que muchas consideraciones ambientales están limitadas por el interés de los promotores inmobiliarios y del mercado, en donde el precio del suelo y los procesos de especulación son fundamentales.

Asimismo, la planeación de vivienda y ciudad debe tener presentes la accesibilidad, la calidad urbana del entorno, los servicios, la movilidad y las infraestructuras adecuadas para la calidad de vida, así como componentes culturales, en particular cuando se trata de vivienda rural o para sectores sociales particulares ${ }^{11}$. Estos rasgos no son visibles en un contexto en donde se hayan fomentado procesos de compactación como los propuestos por el urbanismo ecológico, más cuando se recomiendan edificaciones heterogéneas un tanto

10 Por ejemplo, los ecobarrios de Trinitat Nova en Barcelona, de Sarrigurren en Navarra, San Francisco Javier, Plata y Castañar y Nuestra Señora de los Ángeles en Vallecas, Madrid, el proyecto Sociópolis en Valencia y proyectos turísticos como el ParcBIT en Mallorca, Islas Baleares y el concurso de urbanización bioclimática en Tenerife, Islas Canarias. 11 Por ejemplo la vivienda social para habitantes de escasos recursos y en condiciones de vulnerabilidad, especialmente en países en vías de desarrollo. 
Figura 2

Ecobarrio de Logroño

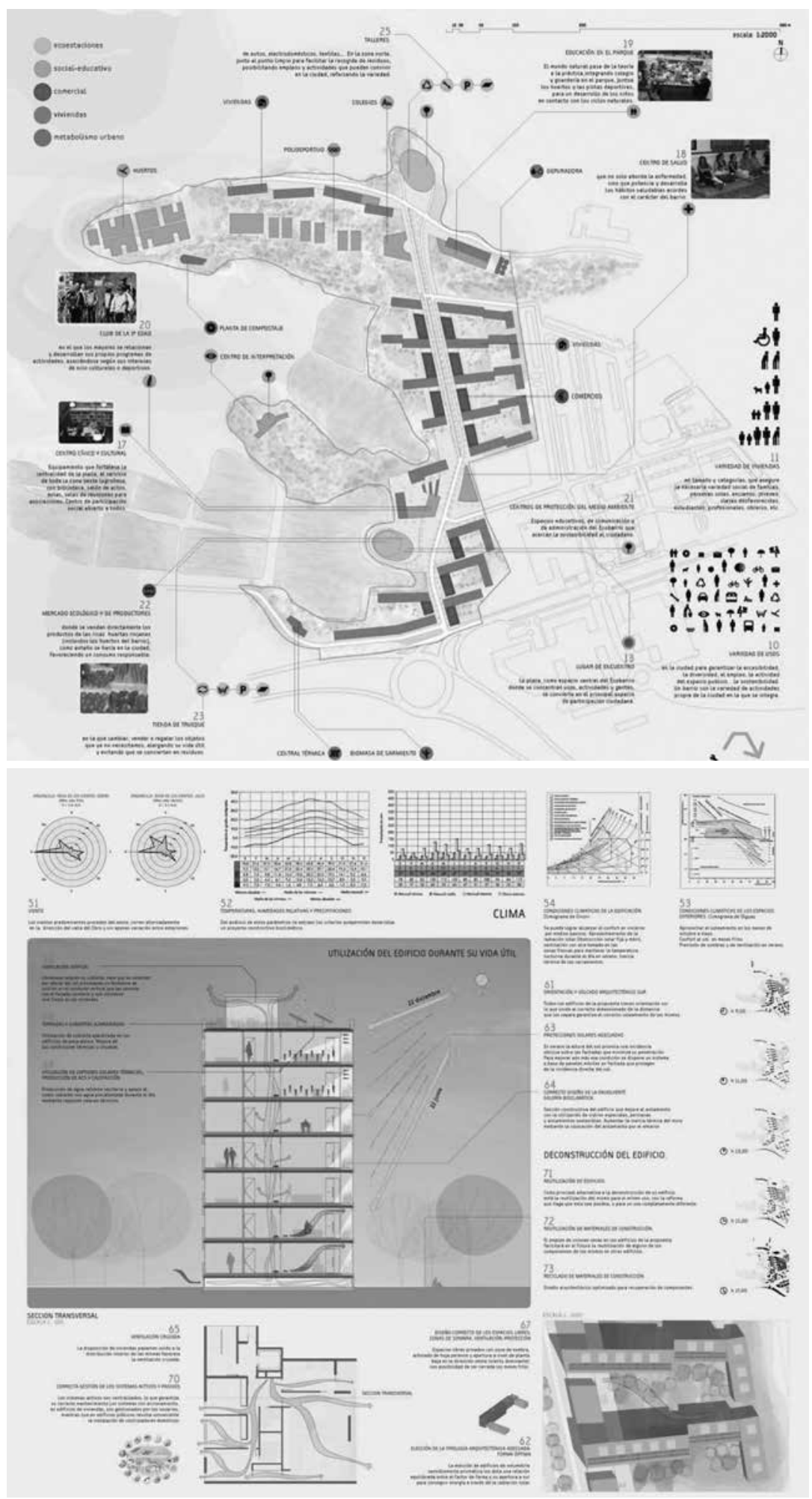


dispersas y la percepción de densidades subjetivas al interior del edificio ${ }^{12}$ es un fenómeno no muy estudiado.

El escenario o la imagen física de ciudad que queremos, la atención al mercado del suelo en el marco de la densificación y redensificación y la incorporación de elementos socioculturales en el diseño constituyen la prospectiva a trabajar en la consolidación de mejores viviendas de alta densidad en ciudades compactas y con principios de urbanismo ecológico.

\section{Bibliografía}

"Los cinco imprescindibles de una vivienda sostenible". (1 de abril de 2013). El Mundo. Recuperado de http://www.elmundo.es/elmundo/2013/04/01/ suvivienda/1364832630.html

Delgado, L. (2005). Urbanismo y arquitectura ecológicos: los territorios de la ecología humana. Recuperado de http://habitat.aq.upm.es/boletin/n35/aldel.html

Feria, J. y Ramos, J. (2009). Funciones ecológicas del espacio libre y planificación territorial en ámbitos metropolitanos: perspectivas teóricas y experiencias recientes en el contexto español. Scripta Nova, XIII(299). Recuperado de http:// www.ub.edu/geocrit/sn/sn-299.htm

Giraldo, F. (2004). Hábitat y desarrollo humano. Bogotá: Cenac.

Haramoto, E. (1983). Políticas de vivienda social: experiencias chilenas de las tres últimas décadas. En J. Mac Donald y M. Collados (eds.), Vivienda social: reflexiones y experiencias (pp. 75-151). Santiago: Corporación de Promoción Universitaria.
Higueras, E. (2006). Urbanismo bioclimático. Barcelona: Gustavo Gili.

Higueras, E. (2009). Buenas prácticas en arquitectura y urbanismo para Madrid: criterios bioclimáticos y de eficiencia energética. Madrid: Área de Gobierno de Urbanismo y Vivienda del Ayuntamiento de Madrid.

Iglesias, P. (2010). Introducción a la vivienda sostenible o vivienda sostenible para el moderno Prometeo. $A X A$. Recuperado de http://www.uax. es/publicacion/introduccion-a-la-vivienda-sostenible.pdf

López, B. y Marzo R. (s. f.). Propuesta de ecobarrio en Logroño: recuperando los valores de la ciudad tradicional. Recuperado de http://urban-e.aq.upm.es/miscelanea/view/propuesta-deecobarrio-en-logro-o-recuperando-los-valoresde-la-ciudad-tradicional/full

Luque, J. (septiembre de 2011). Insuficiencia de la ciudad compacta. Conferencia presentada en el VII Congreso iberoamericano de urbanismo, región y calidad urbana, Pamplona, Asociación Española de Técnicos Urbanistas.

Molini, F. y Salgado, M. (2012). Los impactos ambientales de la ciudad de baja densidad en relación con los de la ciudad compacta. Biblio $3 \mathrm{~W}$, XVII(958). Recuperado de http://www.ub.edu/ geocrit/b3w-958.htm

Navarro, J. y Ortuño, A. (2011). Aproximación a la génesis de la contribución de la densidad en la noción de "ciudad compacta". EURE, 37(112), 23-41.

Real Academia Española. (2014). Multifamiliar. En Diccionario de la lengua española. Recuperado de http://dle.rae.es/?id=DgIqVCc 
Rincón, P. (2006). Bogotá y sus modalidades de ocupación del suelo: análisis de los procesos de re-densificación. Bogotá: Universidad Nacional de Colombia.

Ruano, M. (1999). Ecourbanismo: entornos humanos sostenibles, 60 proyectos. Barcelona: Gustavo Gili.

Rueda, S. (1997). La ciudad compacta y diversa frente a la conurbación difusa. Recuperado de http://habitat.aq.upm.es/cs/p2/a009.html

Rueda, S. (2011a). El urbanismo ecológico. Recuperado de http://urban-e.aq.upm.es/articulos/ ver/el-urbanismo-ecol-gico/completo
Rueda, S. (2011b). El urbanismo ecológico. En J. Ezquiaga y L. González (eds.), Transformaciones urbanas sostenibles (pp. 234-267). Madrid: Universidad Internacional Menéndez Pelayo.

Verdaguer, C. (2000). De la sostenibilidad a los ecobarrios. Documentación social (119), 59-78.

Yory, C. (2004). Ciudady sustentabilidad. Marco general y descripción de la problemática. Bogotá: Universidad Piloto de Colombia. 